\title{
Editorial
}

\section{Efecto de la salud mental en los desenlaces clínicos de los pacientes con cáncer urológico}

\section{Mental health effect on clinical outcomes of patients with urological cancer}

\author{
Herney Andrés García-Perdomo ${ }^{10}$ Isabel Carolina Osorio Lasso ${ }^{2}$ \\ 1 Sección de Urología, Departamento de Cirugía, Escuela de Medicina, \\ Universidad del Valle, Cali, Colombia \\ ${ }^{2}$ Instituto Chileno de Psicoterapia, Santiago de Chile, Chile
}

Urol Colomb 2020;29:111-112.

El abordaje integral del paciente oncológico incluye un sinnúmero de parámetros clínicos, que normalmente no se tienen en cuenta durante el diagnóstico y el tratamiento. La mayoría de las ocasiones se minimiza la salud mental del paciente y de su familia, dejando sin atender aspectos emocionales que pueden tener un efecto en los desenlaces clínicos de estos.

La evidencia de una respuesta neuroendocrina dada por los estados del ánimo lleva a concluir que se requiere tomar herramientas de contención emocional como parte importante del diagnostico, tratamiento, seguimiento y rehabilitación de un paciente oncológico. ${ }^{1}$

La depresión y la ansiedad, así como otros trastornos mentales, usualmente se identifican en aquellos pacientes que se encuentran en tamizaje o quienes reciben un diagnóstico de cáncer urológico. Además, la respuesta adaptativa va condicionada por la cultura, sus recursos emocionales y múltiples aspectos neurobiológicos: la reacción individual a un estrés crónico y prolongado, la activación sostenida del Sistema Nervioso Autónomo y del eje hipotálamo-hipófisis-adrenal. La exposición a lo previamente descrito impacta a cada persona de diferente manera, pudiendo afectar la respuesta y la magnitud del cuadro clínico. ${ }^{2}$

El enfrentar un proceso inicial de estudios y luego un diagnóstico de cáncer, genera diferentes reacciones que van desde la negación y desorientación hasta el pánico y la rabia. El paciente percibe una amenaza por la perdida de su salud, además de la vulnerabilidad corporal por los cambios que vendrán y la incorporación a su nueva cotidianidad, que incluirá alteraciones físicas, sociales, económicas y el temor por una recurrencia tumoral.

Esta cantidad de emociones y alteraciones mentales pueden llevar a tener efectos negativos en los desenlaces clínicos de los pacientes. Con respecto a cáncer de vejiga, se ha encontrado que un estado mental alterado se asocia con un riesgo incrementado de complicaciones, incluyendo un tiempo menor de progresión de la enfermedad, después de cistectomía radical. ${ }^{3,4}$ Por otro lado, aquellos pacientes sometidos a neovejigas ortotópicas quienes contaron con un acompañamiento psicológico mejoraron su tiempo de retorno a las actividades. ${ }^{1,5}$ Con respecto al cáncer de pene, se encontró que los pacientes que presentaban depresión antes del procedimiento quirúrgico tenían un tiempo mayor de recuperación de sus heridas. Además, tenían sensación de minusvalía, depresión, ansiedad e incapacidad de conectar con los demás. ${ }^{6}$

En el caso del cáncer urogenital se identifican múltiples factores de riesgo para exacerbación de patología mental por el gran compromiso a nivel del funcionamiento sexual, los importantes cambios corporales que desencadenan percepciones de limitación en la funcionabilidad tanto corporal como emocional. ${ }^{7}$

La evaluación de todos los factores que intervienen en la decisión del mejor tratamiento debe ser acompañada de un profesional en salud mental, contribuyendo así, positivamente, en la respuesta al proceso elegido; sea curativo o paliativo. Desde allí, aportar en la calidad y permanencia de resultados benéficos. El acompañamiento psicoterapéutico temprano, humanizado y paralelo al proceso farmacológico y/o quirúrgico debe ser una conducta permanente, que garantice un proceso integral. De esta manera, se contribuye al aprendizaje de lo que implica la pérdida de una "normalidad". El profesional en salud mental esta en capacidad de prevenir e identificar entidades preexistentes que pueden empeorar el pronóstico y la rehabilitación del paciente. ${ }^{1}$

Establecer un diagnóstico y un tratamiento precoces, junto al acompañamiento y la contención por parte de un equipo multidisciplinario en el que se integre al profesional
Address for correspondence Herney Andrés García-Perdomo, MD MSc, EdD, PhD, FACS, Departamento de Cirugía/Urología, Escuela de Medicina, Universidad del Valle, Cali, Colombia

(e-mail: editorrevista@scu.org.co).
DOI https://doi.org/ 10.1055/s-0040-1716514. ISSN 0120-789X. e ISSN 2027-0119.

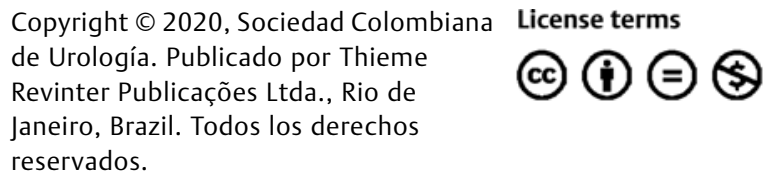

reservados. 
en salud mental, puede mejorar la calidad de vida y la buena respuesta a su padecimiento. Aún faltan estudios que soporten el efecto que tiene una adecuada salud mental en los desenlaces clínicos de éstos pacientes; sin embargo, en la práctica clínica se evidencia la necesidad de intervenir tempranamente en la salud mental del paciente como factor protector frente al desenlace de su condición médica y de la misma salud mental ${ }^{7}$

\section{Referencias}

1 Pham X, Torres H, Sharma P. Mental health implications in bladder cancer patients: A review. Urol Oncol 2018;0:1-11

2 Tafet GE, Nemeroff CB. The links between stress and depression: Psychoneuroendocrinological, genetic, and environmental interactions. J Neuropsychiatry Clin Neurosci 2016;28(02):77-88 https://pubmed.ncbi.nlm.nih.gov/26548654/cited2020Aug9 [Internet]
3 Sharma P, Henriksen CH, Zargar-Shoshtari K, et al. Preoperative patient reported mental health is associated with high grade complications after radical cystectomy. J Urol 2016;195(01): 47-52

4 Palapattu GS, Bastian PJ, Slavney PR, et al. Preoperative somatic symptoms are associated with disease progression in patients with bladder carcinoma after cystectomy. Cancer 2004;101(10): 2209-2213

5 Yang M, Wang H, Wang J, Ruan M. Impact of invasive bladder cancer and orthotopic urinary diversion on general health-related quality of life: An SF-36 survey. Mol Clin Oncol 2013;1(04):758-762

6 Witty K, Branney P, Evans J, Bullen K, White A, Eardley I. The impact of surgical treatment for penile cancer - patients' perspectives. Eur J Oncol Nurs 2013;17(05):661-667. Doi: 10.1016/j.ejon.2013.06.004 [Internet]

7 Purushotham A, Bains S, Lewison G, Szmukler G, Sullivan R. Cancer and mental health-a clinical and research unmet need. Ann Oncol 2013;24(09):2274-2278https://www.annalsofonco logy.org/article/S0923-7534(19)36946-7/fulltext cited2020Aug9 [Internet] 